\title{
INTEGRAL constraints on the Galactic hard X-ray background from the Milky Way anticenter ${ }^{\star} \star \star \star$
}

\author{
R. Krivonos ${ }^{1,2}$, S. Tsygankov ${ }^{1,2,3,4}$, M. Revnivtsev ${ }^{2}$, S. Sazonov ${ }^{2}$, E. Churazov ${ }^{1,2}$, and R. Sunyaev ${ }^{1,2}$ \\ 1 Max-Planck-Institut für Astrophysik, Karl-Schwarzschild-Str. 1, 85740 Garching bei München, Germany \\ e-mail: krivonos@mpa-garching.mpg.de \\ 2 Space Research Institute, Russian Academy of Sciences, Profsoyuznaya 84/32, 117997 Moscow, Russia \\ 3 Finnish Centre for Astronomy with ESO (FINCA), University of Turku, Väisäläntie 20, 21500 Piikkiö, Finland \\ 4 Astronomy Division, Department of Physics, 90014 University of Oulu, Finland \\ Received 8 September 2011 / Accepted 16 November 2011
}

\section{ABSTRACT}

\begin{abstract}
We present results of a study of the Galactic ridge X-ray emission (GRXE) in hard X-rays with the IBIS telescope onboard INTEGRAL in the region near the Galactic anticenter (GA) at $l=155^{\circ}$. We assumed a conservative $2 \sigma$ upper limit on the flux from the GA in the 25-60 keV energy band of $1.25 \times 10^{-10} \mathrm{erg} \mathrm{s}^{-1} \mathrm{~cm}^{-2}(12.8 \mathrm{mCrab})$ per IBIS field of view, or $6.6 \times 10^{-12} \mathrm{erg} \mathrm{s}^{-1} \mathrm{~cm}^{-2}$ $(0.7 \mathrm{mCrab})$ per degree longitude in the $135^{\circ}<l<175^{\circ}$ region. This upper limit exceeds the expected GRXE intensity in the GA direction by an order of magnitude, given the near-infrared (NIR) surface brightness of the Milky Way in this region and the standard hard X-ray-to-NIR intensity ratio for the GRXE, assuming stellar origin. Based on the CGRO/EGRET surface brightness of the Galaxy above $100 \mathrm{MeV}$ as a tracer of the cosmic-ray (CR) induced gamma-ray background, the expected GRXE flux in GA exceeds the measured $2 \sigma$ upper limit by a factor of 4 . Therefore, the non-detection of hard X-ray emission from the GA does not contradict the stellar nature of the GRXE, but is inconsistent with CR origin.
\end{abstract}

Key words. Galaxy: structure - X-rays: diffuse background

\section{Introduction}

The stellar origin of the Galactic hard X-ray background, better known as the Galactic ridge X-ray emission (GRXE), has recently been strongly supported by morphological/spectral studies with the RXTE and INTEGRAL observatories (Revnivtsev et al. 2006; Krivonos et al. 2007a; Türler et al. 2010), spectral studies with Suzaku (Ebisawa et al. 2008; Yamauchi et al. 2009) and direct X-ray source counts with Chandra (Revnivtsev et al. 2009, 2011). The GRXE does not arise from the interaction of cosmic rays with the interstellar medium, as was believed before, but is associated with the (predominantly old) stellar population of the Galaxy, namely with hard X-ray emission from accreting white dwarfs and coronaly active stars. It was demonstrated (Revnivtsev et al. 2006; Krivonos et al. 2007a) that the GRXE intensity closely follows near-infrared (NIR) surface brightness over the Milky Way, which is a known tracer of stellar mass.

Galactic diffuse emission in a region far away from the Galactic center (GC) was studied by Worrall et al. (1982) with the A2 experiment onboard the HEAO1 satellite. The observed 2-10 keV emission was consistent with an radial exponential disk with a half-thickness of $\sim 240 \mathrm{pc}$. It was pointed out that unresolved emission likely comes from discrete point sources and does not have a diffuse origin. In the hard X-ray domain GRXE was studied by Skibo et al. (1997) using OSSE observations at longitude $l=95^{\circ}$. The observed emission between

\footnotetext{
* Based on observations with INTEGRAL, an ESA project with instruments and science data centre funded by ESA member states (especially the PI countries: Denmark, France, Germany, Italy, Switzerland, Spain), Czech Republic and Poland, and with the participation of Russia and the USA.

$\star \star$ Appendices are available in electronic form at

http://www. aanda.org
}

50 and $600 \mathrm{keV}$ was suspected to contain a significant contribution from bright discrete sources because of the wide collimated field of view $\left(\sim 11^{\circ} .4 \times 3.8\right)$, but a major part of the detected flux was interpreted as interstellar emission from non-thermal electrons (see also e.g. Valinia et al. 2000; Valinia \& Tatischeff 2001). Today, thanks to the unique possibilities of INTEGRAL gamma-ray telescopes, we can directly study the Galactic diffuse background in any parts of the Galaxy without dealing with significant source contamination.

Given the distribution of $4.9 \mu \mathrm{m}$ NIR intensity over the Galaxy as measured by the COBE/DIRBE experiment, the GRXE is not expected to be detectable in the Galactic anticenter (GA) because of the low NIR surface brightness in this region. Nevertheless, an explicit demonstration that the GRXE is not observed from a Galactic region of low stellar density, such as the GA, would substantiate the stellar paradigm of the GRXE even more. Placing tight constraints on the hard X-ray flux from the GA region is also important for calibrating future studies of the GRXE in the central parts of the Galaxy.

\section{Observations}

We used data from the ISGRI detector, the first layer of the IBIS coded-mask telescope (Ubertini et al. 2003), onboard the INTEGRAL gamma-ray observatory (Winkler et al. 2003). ISGRI operates at energies above $\sim 20 \mathrm{keV}$, with the sensitivity rapidly decreasing above $100 \mathrm{keV}$. IBIS has a relatively wide field of view $\left(\sim 28^{\circ} \times 28^{\circ}\right.$ at zero response), which allows one to measure weak diffuse emission fluxes by using the telescope as a collimated instrument.

To study the GRXE in the GA region, we used special INTEGRAL observations, part of a series of so-called Galactic 
Table 1. INTEGRAL observations used for the GRXE study.

\begin{tabular}{lcc}
\hline \hline \multicolumn{3}{c}{ Latitude scan at $l=155^{\circ}, 2010$} \\
\hline Observation field & Orbits & ScWs \\
\hline$\ldots$ & 960,961 & $2-94,11-97$ \\
$\ldots$ & 962,963 & $12-112,1-97$ \\
$\ldots$ & 964,965 & $13-110,2-67$ \\
$\ldots$ & 966 & $23-56$
\end{tabular}

$569 \mathrm{ScWs}$, total nominal exposure: $1 \mathrm{Ms}$.

\begin{tabular}{lc}
\hline \multicolumn{2}{c}{ Background model calibration, 2008-2009 } \\
\hline Observation field & Orbits \\
\hline North Ecliptic Pole & $686-689,759-761,824-829$ \\
XMM-LSS & $695,696,701$ \\
Virgo Cluster & $747-754,758,819-820$ \\
Coma Cluster & $821-823$ \\
M82 X-1 & $869-872$ \\
3C273, 3C279 and M87 & $878-880$ \\
& Total nominal exposure: 6 Ms. \\
\hline \multicolumn{2}{c}{ Crab calibration, 2010 } \\
\hline Observation field & Orbits \\
\hline Crab nebula & 902, 903, 966-968, 970 \\
\multicolumn{2}{c}{ Total nominal exposure: 890 ks. } \\
\hline
\end{tabular}

latitude scans (GLS). This program is based on consecutive observations made along the Galactic latitude in the range $\pm 30^{\circ}$ with a step of $2^{\circ}$. This strategy allows one to make independent snapshot measurements of the instrumental background at mid latitudes, where the GRXE is expected to be negligible, along with an actual GRXE observation near the Galactic plane. It is crucial that the instrumental background, which is usually high in hard X-ray observations, does not exhibit strong variability during an individual GLS lasting $\sim 8 \mathrm{~h}$.

There are two positions in the GA region observed with GLSs: ongoing observations at $l=215^{\circ}$ (PI: Tsygankov) and a completed program at $l=155^{\circ}$ with a total exposure of $1 \mathrm{Ms}$ (PI: Krivonos). In the present study we only used the completed GLS observations at $l=155^{\circ}$ performed in August-September 2010, see Table 1 for details. After screening the whole data set following the procedure described in Krivonos et al. (2007a), hereafter K07, we were left with 525 out of 569 short ( $2 \mathrm{ks})$ individual INTEGRAL observations, called scientific windows ( $S c W \mathrm{~s}$ ), for the subsequent scientific analysis. To model background variations we used public data of high-latitude observations (Table 1).

\section{Analysis}

We mainly followed the approach described in K07. Our study of the GRXE is based on the capability of the IBIS telescope to separate the contributions of point sources from the background count rate.

The ISGRI detector shadowgram that was accumulated during individual INTEGRAL observation in a given energy range was cleaned from source fluxes using the known pattern of the IBIS mask. The remaining shadowgram contains the following components: (i) variable instrumental background; (ii) the isotropic cosmic X-ray background (CXB); and (iii) the GRXE. Throughout the analysis we assumed the CXB flux as a constant part of the instrumental background. The possible influence of the CXB variance on the GRXE measurement is considered in Appendix B. Because the GRXE is extended over the sky, it cannot be directly resolved with the IBIS mask. The only way to estimate the GRXE flux is to determine the difference between the observed collimated detector count rate that is cleaned from sources and the assumed instrumental background. To this end, we define a background model in Appendix A that predicts the background count rate during a given GRXE observation. The background model is adjusted very precisely using mid-latitude snapshots of the background performed shortly before and after a given GRXE observation, which is the main concept of the GLS.

\subsection{Detector shadowgram}

Because IBIS is a coded aperture imaging telescope, the sky is projected onto the detector plane through the transparent and opaque elements of the mask mounted above the detector plane. We produced the ISGRI detector shadowgram for every $S c W$ as described in $\mathrm{K} 07$. We used the $25-60 \mathrm{keV}$ working energy band because of the known evolution of the low-energy threshold of the ISGRI detector and because the GRXE is expected to be weak above $\sim 50 \mathrm{keV}$ owing to the high-energy cut-off in the GRXE spectrum (Krivonos et al. 2007a; Revnivtsev et al. 2006).

\subsection{Sky map}

The sky reconstruction is based on deconvolution of the detector shadowgram with a known mask pattern. We implemented the IBIS/ISGRI sky reconstruction method described in our previous publications (Revnivtsev et al. 2004; Krivonos et al. 2005, $2007 a, b)$. For the basic idea we refer the reader to the papers by Fenimore \& Cannon (1981) and Skinner et al. (1987). Every sky image was additionally cleaned from systematic noise as described in Krivonos et al. (2010a). The resulting sky image mosaic is shown in Fig. 1.

The survey area as a function of flux for sources with $S / N>$ 5 is shown in Fig. 2. The minimum detectable flux in the central part of the field is $2.4 \times 10^{-11} \mathrm{erg} \mathrm{s}^{-1} \mathrm{~cm}^{-2}$ (or $2.5 \mathrm{mCrab}$ ). The survey area reaches its geometric limit of $3650 \mathrm{deg}^{2}$ for $f>$ $4.9 \times 10^{-9} \mathrm{erg} \mathrm{s}^{-1} \mathrm{~cm}^{-2}(500 \mathrm{mCrab}), 50 \%$ of this area has a sensitivity better than $8.1 \times 10^{-11} \mathrm{erg} \mathrm{s}^{-1} \mathrm{~cm}^{-2}$ ( $\left.8.3 \mathrm{mCrab}\right)$.

We performed a search for sources as excesses in the sky mosaic (Fig. 1) convolved with a Gaussian representing the effective instrumental PSF. The detection threshold was estimated assuming Gaussian noise of the pixel values as follows. The total area of the sky image (Fig. 1) is 3650 squared degrees, and taking the IBIS telescope angular resolution of $12^{\prime}$ into account, we gathered $\sim 9.12 \times 10^{4}$ independent pixels. With this consideration, we set the source detection threshold to $5 \sigma$, allowing at most one false detection for pure Gaussian noise.

The signal-to-noise ratio distribution of pixels shown in Fig. 3 has the expected Gaussian shape. However, one notices some systematic excess at the negative side. Nevertheless, the source detection threshold of $5 \sigma$, estimated above, separates the noise and source dominated pixel domains.

The list of point sources is presented in Table 2. We attribute two marginally detected, previously unknown sources to the systematic noise. IGR J08135+5655 is not detected in the sky mosaic of the slightly broader energy band $20-60 \mathrm{keV}$, and the region around IGR J01532+2612 is affected by systematic noise at the edge of the sky mosaic.

The list of sources in Table 2, except for two IGR's mentioned above, was used in the iterative source removal (IROS) procedure that we applied to every ISGRI detector shadowgram (e.g. Krivonos et al. 2010a). This procedure introduces additional uncertainty to the background model (Appendix C), but allows one to trace source variability. For example, the known Be/X-ray binary RX J0440.9+4431 (LS V +44 17) was in a strong outburst during the observations (Krivonos et al. 2010c; Tsygankov et al. 2011). Figure 4 shows $25-60 \mathrm{keV}$ detector light 
R. Krivonos et al.: Constraints on the ridge emission from the Milky Way anticenter

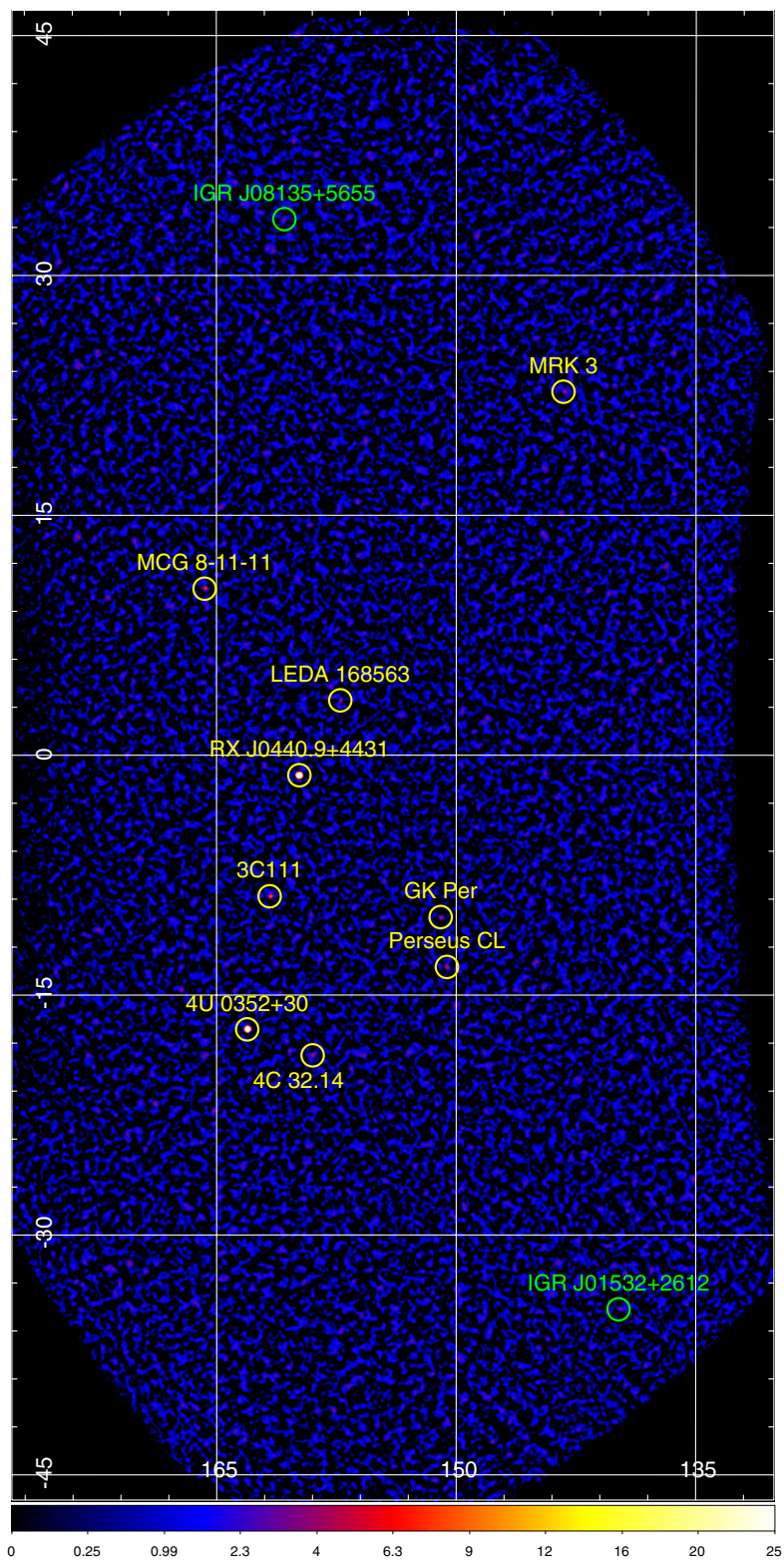

Fig. 1. IBIS/ISGRI sky image of the $1 \mathrm{Ms}$ observation of the GA region at $l=155^{\circ}$ produced in the $25-60 \mathrm{keV}$ energy range. The color table of the image represents pixel values in the range $0-25$ calculated as the square root of the significance. The cataloged and newly detected sources are labeled in yellow and green, respectively.

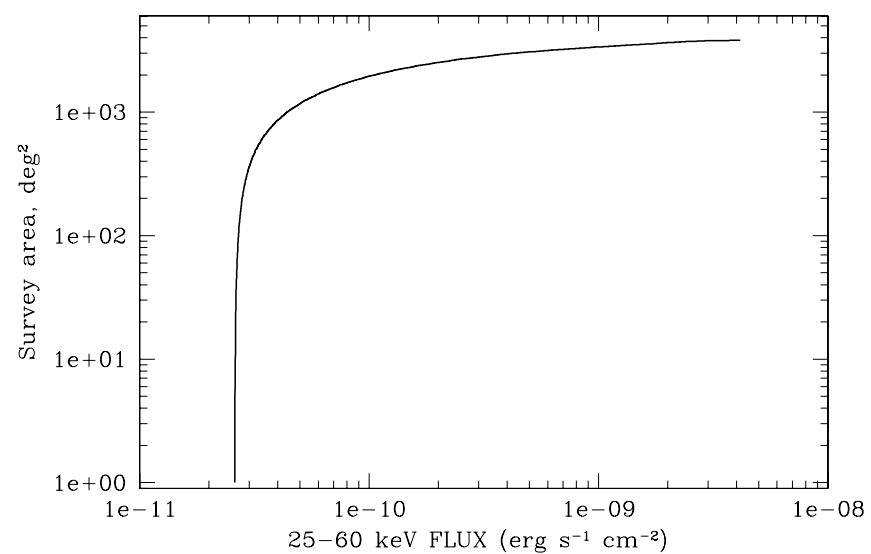

Fig. 2. Covered area of the survey as a function of flux for sources with $S / N>5$.

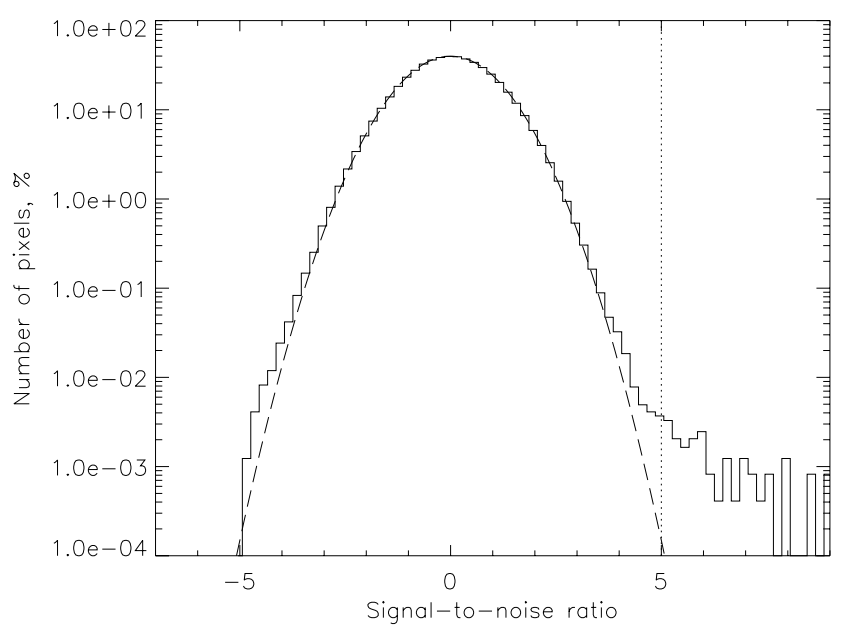

Fig. 3. Signal-to-noise ratio distribution of pixels in the $1 \mathrm{Ms}$ GLS observation at $l=155^{\circ}$. The dashed line represents the normal distribution with unit variance and zero mean. The accepted threshold of source detection $(5 \sigma)$ is shown by the dotted line. The plot is truncated at $\sigma=9$ for convenience.

Table 2. List of sources significantly $(\geq 5 \sigma)$ detected on the sky mosaic (Fig. 1).

\begin{tabular}{|c|c|c|c|}
\hline Name & $\begin{array}{l}\text { lon. } \\
\text { deg }\end{array}$ & $\begin{array}{l}\text { lat. } \\
\text { deg }\end{array}$ & $\begin{array}{c}F_{25-60 \mathrm{keV}} \\
\text { mCrab }\end{array}$ \\
\hline $4 \mathrm{U} 0352+30^{a}$ & 163.08 & -17.14 & $39.42 \pm 0.69$ \\
\hline $\mathrm{RX} \mathrm{J0440.9+4431^{a }}$ & 159.82 & -1.26 & $26.12 \pm 0.53$ \\
\hline $3 \mathrm{C} 111^{b}$ & 161.68 & -8.83 & $5.32 \pm 0.60$ \\
\hline MCG 8-11-11 ${ }^{b}$ & 165.74 & 10.41 & $7.27 \pm 0.86$ \\
\hline $4 \mathrm{C} 32.14^{c}$ & 158.99 & -18.77 & $3.15 \pm 0.56$ \\
\hline LEDA $168563^{b}$ & 157.26 & 3.43 & $2.76 \pm 0.49$ \\
\hline MRK $3^{b}$ & 143.29 & 22.72 & $7.23 \pm 1.33$ \\
\hline Perseus $\mathrm{CL}^{d}$ & 150.58 & -13.25 & $3.06 \pm 0.58$ \\
\hline IGR J01532+2612 & 139.82 & -34.64 & $20.31 \pm 3.91$ \\
\hline IGR J08135+5655 & 160.75 & 33.53 & $7.09 \pm 1.38$ \\
\hline GK Per $^{e}$ & 150.96 & -10.12 & $2.82 \pm 0.56$ \\
\hline
\end{tabular}

Notes. The newly detected sources are highlighted in bold. The $68 \%$ confidence interval for estimated sky coordinates depends on source significance: $2.1^{\prime}, 1.5^{\prime}$, and $<0.8^{\prime}$ for $5-6,10$, and $>20 \sigma$, respectively (Krivonos et al. 2007b). ${ }^{(a)} \mathrm{HMXB},{ }^{(b)} \mathrm{AGN},{ }^{(c)} \mathrm{QSO},{ }^{(d)}$ Cluster of Galaxies, ${ }^{(e)} \mathrm{CV}$.

curve, cleaned from the source contribution, and ready for further analysis.

\section{Results}

Using the background model (Appendix A) we obtained for each $\mathrm{ScW}$ the predicted detector count rate, as shown in Fig. 5. The detailed view of the observed and predicted detector light curves and their residuals during a spacecraft orbit 964 is shown in Fig. 6. We denoted regions of background measurement $(|b|>=$ $\left.20^{\circ}\right)$ in blue, and actual GRXE observations $\left(|b|<20^{\circ}\right)$ in red. Figure 6 shows that the background behavior in an individual orbit can be captured only with fast scanning observations such as GLSs. The background model, with low intrinsic scatter, exactly follows the observed detector rate, and the scatter of residuals (lower panel of Fig. 6) is comparable to the statistical uncertainty of the IROS procedure (Appendix C).

Using the entire GLS data set at $l=155^{\circ}$, we averaged residuals over Galactic latitude, as shown in Fig. 7 by red points. The latitude profile does not show any significant excess in the 


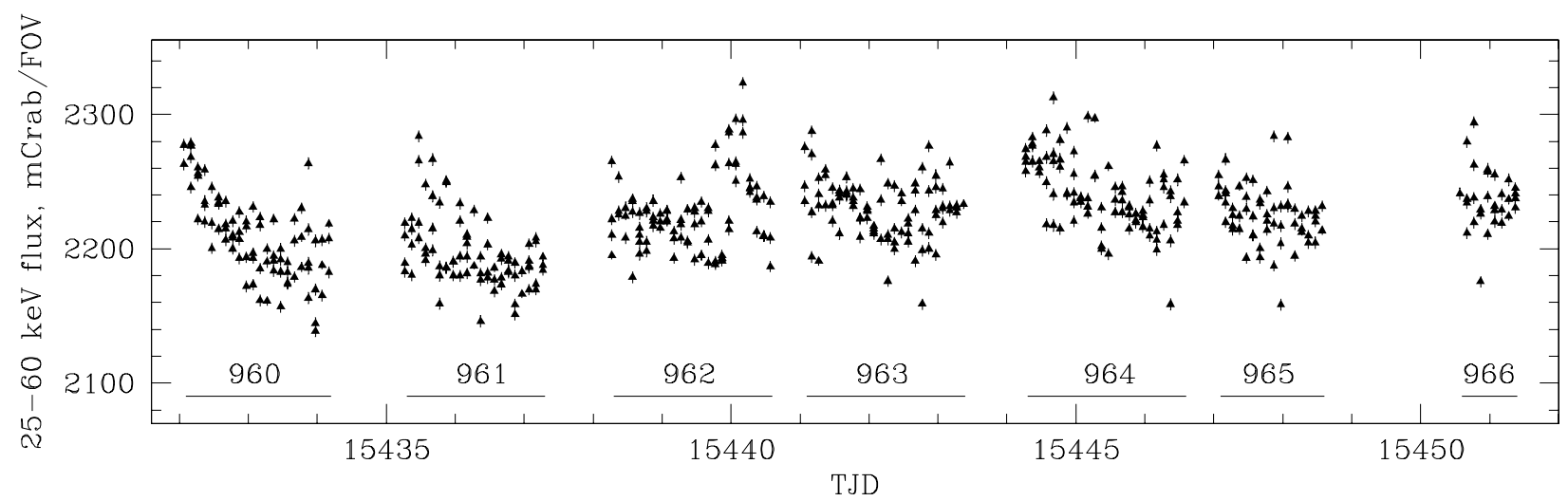

Fig. 4. Detector count rate of the individual $S c W$ s after subtracting flux contribution from point sources during the GLS program at $l=155^{\circ}$. The data points contain pure statistical errors. The labeled horizontal intervals denote different spacecraft orbits.

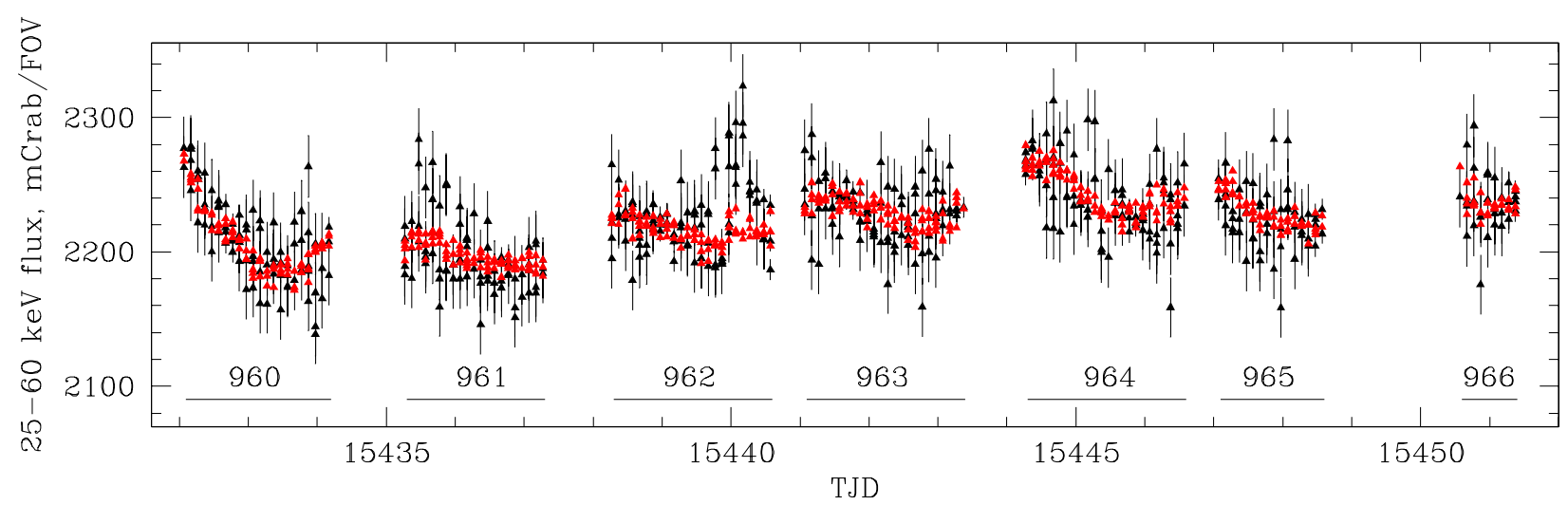

Fig. 5. Detector count rate (black) of the individual $S c W$ s as shown in Fig. 4. The systematic uncertainty of the source removal procedure (Appendix C) was added to the statistical errors of each point. The red points represent the count rate predicted by the background model.

Galactic plane region at $b=0^{\circ}$. As expected, the GRXE associated with the old stellar population is not detected in the GA. The $1 \sigma$ upper limit on the GRXE flux in the $|b|<5^{\circ}$ latitude range, which roughly corresponds to the IBIS fully coded FOV, is $\sim 2.8 \mathrm{mCrab}$, or $\sim 6.4 \mathrm{mCrab}$ taking systematic uncertainties into account. We later refer to a $2 \sigma$ upper limit of $\sim 12.8 \mathrm{mCrab}$. One can convert the achieved upper limit to more convenient units using the effective solid angle of the IBIS telescope $\sim 286 \mathrm{deg}^{2}$ and taking into account that the GRXE is much less extended in the Galactic latitude than the average cross-section of IBIS FOV. For instance, the $2 \sigma$ upper limit on the GRXE flux at $l=155^{\circ}$ per unit Galactic longitude is $0.7 \mathrm{mCrab} \mathrm{deg}^{-1}$.

One can test the GRXE non-detection in the GA for consistency with stellar and truly diffuse GRXE origins. To this end, we compared the observed drop of the hard X-ray flux from the GC to the GA region (per IBIS FOV) with the corresponding change of the intensity of a given tracer. We used the COBE/DIRBE $4.9 \mu \mathrm{m}_{\text {data }}{ }^{1}$ as a tracer of stellar mass, and the EGRET gamma-ray background map above $100 \mathrm{MeV}$ as a tracer of the cosmic-ray induced gamma-ray background. The EGRET background intensity drops from the GC to the GA by a factor of $\sim 3$. Therefore, using the conservative estimate of the GRXE flux in the GC from $\mathrm{K} 07$ of $150 \pm 15 \mathrm{mCrab}$, we expect the corresponding flux from the GA to be $\sim 50 \mathrm{mCrab}$. This is definitely not observed according to Fig. 7. In contrast, there is a factor of 270 drop in the NIR $4.9 \mu \mathrm{m}$ intensity from $2.7 \times 10^{-5} \mathrm{erg} \mathrm{s}^{-1} \mathrm{~cm}^{-2}$ to $10^{-7} \mathrm{erg} \mathrm{s}^{-1} \mathrm{~cm}^{-2}$. This implies a GRXE flux from the GA of $0.4 \mathrm{mCrab}$ at $25-60 \mathrm{keV}$, which is

\footnotetext{
${ }^{1} \mathrm{COBE} / \mathrm{DIRBE} 4.9 \mu \mathrm{m}$ intensity map was corrected for the interstellar reddening and mean background level measured in high-latitude regions, as described in $\mathrm{K} 07$.
}

consistent with the derived upper limit of $12.8 \mathrm{mCrab}$. This is illustrated in Fig. 8, where the COBE/DIRBE and EGRET longitude profiles are renormalized to the hard X-ray flux observed from the GC. We conclude that the non-detection of the GRXE from the GA is consistent with the stellar mass distribution of the Galaxy traced by NIR maps, rather than with the cosmic-ray induced gamma-ray background seen by EGRET.

\section{Conclusions}

1) Using the $1 \mathrm{Ms}$ observations of the GA at $l=155^{\circ}$ with the INTEGRAL observatory, performed in the special GLS mode, we did not detect the GRXE in the 25$60 \mathrm{keV}$ energy band and set a conservative $2 \sigma$ upper limit of $1.25 \times 10^{-10} \mathrm{erg} \mathrm{s}^{-1} \mathrm{~cm}^{-2}(12.8 \mathrm{mCrab})$ per IBIS FOV or $6.6 \times 10^{-12} \mathrm{erg} \mathrm{s}^{-1} \mathrm{~cm}^{-2} \mathrm{deg}^{-1}\left(0.7 \mathrm{mCrab} \mathrm{deg}^{-1}\right)$ per unit Galactic longitude.

2) The obtained upper limit is consistent with the considerable drop in the NIR $(4.9 \mu)$ intensity observed by COBE/DIRBE and disagrees with the much smaller decrease in the gammaray (above $100 \mathrm{MeV}$ ) background measured by EGRET. Therefore, the non-detection of the GRXE in the GA is consistent with the stellar mass distribution in the Galaxy, which does not contradict the stellar nature of GRXE, but is inconsistent with its CR origin.

3) The developed background model potentially allows one to reach the statistically limited accuracy. However, the final uncertainty of the approach is associated with the source removal procedure, the systematic uncertainty of the method itself, and the CXB variance. Nevertheless, the implemented method along with the special GLS mode of observation is 
R. Krivonos et al.: Constraints on the ridge emission from the Milky Way anticenter
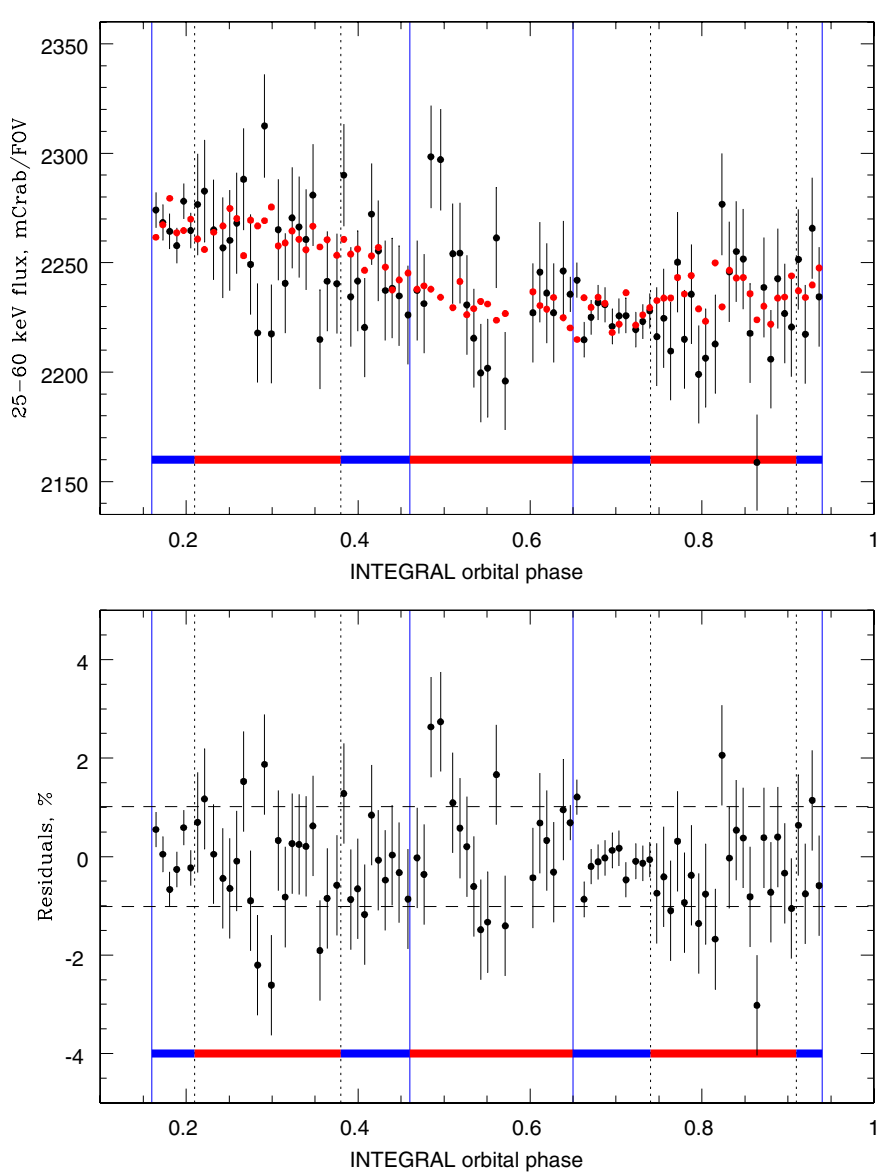

Fig. 6. Upper panel: detailed view of detector count rate (black) during orbit 964 from Fig. 5. The blue and red areas denote observations made at $|b|>=20^{\circ}$ and $|b|<20^{\circ}$, respectively. Bottom panel: residuals after subtracting the model-predicted count rate from the observed count rate. The black dashed lines represent a $1 \sigma$ deviation $(1.02 \%)$ from zero.

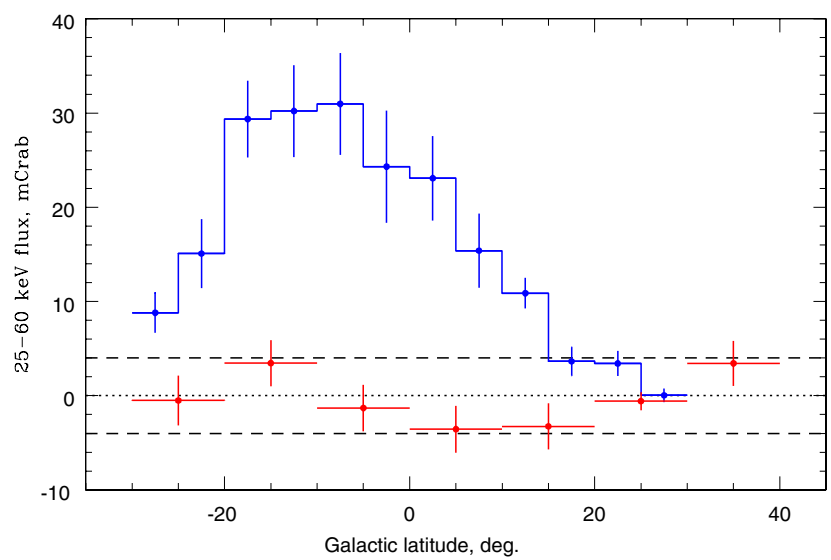

Fig. 7. Source flux contribution (blue) and ISGRI detector background count rate residuals (red) averaged over the Galactic latitude. Error bars of the blue histogram represent rms-deviations of the summed point source fluxes from average in bin.

an optimal approach of modeling the ISGRI background and can be efficiently used for studying the Galactic hard X-ray background.

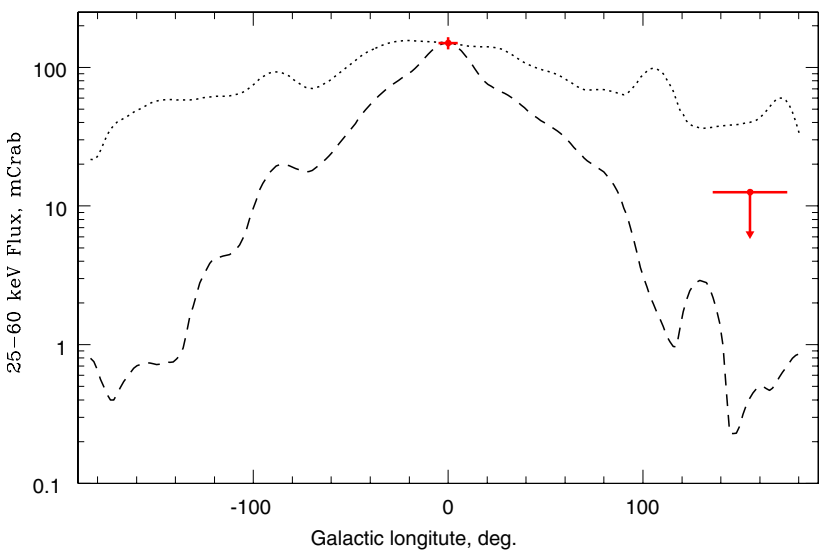

Fig. 8. Galactic longitude profiles of the COBE/DIRBE $4.9 \mu$ m intensity (dashed line) and EGRET background above $100 \mathrm{MeV}$ (dotted line), both normalized to the GRXE flux of $150 \mathrm{mCrab}$ (red point) in the GC. The $2 \sigma$ upper limit corresponds to the GRXE measurement at $l=155^{\circ}$ in the present study.

Acknowledgements. This research was made possible thanks to the unique capabilities of the INTEGRAL observatory. The data used were obtained from the European and Russian INTEGRAL Science Data Centers. The work was supported by the President of the Russian Federation (through the program of support of leading scientific schools, project NSH-5069.2010.2, and grant MD-1832.2011.2), by the Presidium of the Russian Academy of Sciences/RAS (the program "Origin, Structure, and Evolution of Objects of the Universe"), by the Division of Physical Sciences of the RAS (the program "Extended objects in the Universe", OFN-16), by the Russian Basic Research Foundation (grant 10-02-00492-A), State contract 14.740.11.0611, and the Academy of Finland grant 127512 .

\section{References}

Churazov, E., Sunyaev, R., Revnivtsev, M., et al. 2007, A\&A, 467, 529

Ebisawa, K., Yamauchi, S., Tanaka, Y., et al. 2008, PASJ, 60, 223

Fabian, A. C., \& Barcons, X. 1992, ARA\&A, 30, 429

Fenimore, E. E., \& Cannon T. M., 1981, Appl. Opt., 20, 1858

Skinner, G. K., Ponman, T. J., Hammersley, A. P., \& Eyles, C. J. 1987, Astroph. Space Sci., 136, 337

Gruber, D. E., Matteson, J. L., Peterson, L. E., \& Jung, G. V. 1999, ApJ, 520, 124

Hajdas, W., Bühler, P., Eggel, C., et al. 2003, A\&A, 411, L43

Krivonos, R., Vikhlinin, A., Churazov, E., et al. 2005, ApJ, 625, 89

Krivonos, R., Revnivtsev, M., Churazov, E., et al. 2007a, A\&A, 463, 957

Krivonos, R., Revnivtsev, M., Lutovinov, A., et al. 2007b, A\&A, 475, 775

Krivonos, R., Revnivtsev, M., Tsygankov, S., et al. 2010a, A\&A, 519, A107

Krivonos, R., Tsygankov, S., Revnivtsev, M., et al. 2010b, A\&A, 523, A61

Krivonos, R., Tsygankov, S., Lutovinov, A., Turler, M., \& Bozzo, E. 2010c, The Astronomer's Telegram, 2828, 1

Revnivtsev, M., Sunyaev, R., Varshalovich, D., et al. 2004, Astron. Lett., 30, 382 Revnivtsev, M., Sazonov, S., Gilfanov, M., Churazov, E., \& Sunyaev, R. 2006, A\&A, 452, 169

Revnivtsev, M., Molkov, S., \& Sazonov, S. 2008, A\&A, 483, 425

Revnivtsev, M., Sazonov, S., Churazov, E., et al. 2009, Nature, 458, 1142

Revnivtsev, M., Sazonov, S., Forman, W., Churazov, E., \& Sunyaev, R. 2011, MNRAS, 414, 495

Skibo, J. G., Johnson, W. N., Kurfess, J. D., et al. 1997, ApJ, 483, L95

Tsygankov, S., Krivonos, R., \& Lutovinov, A. 2011, MNRAS, submitted

Türler, M., Chernyakova, M., Courvoisier, T. J.-L., et al. 2010, A\&A, 512, A49

Ubertini, P., Lebrun, F., Di Cocco, G., et al. 2003, A\&A, 411, L131

Valinia, A., \& Tatischeff, V. 2001, Exploring the Gamma-Ray Universe, 459, 153

Valinia, A., Kinzer, R. L., \& Marshall, F. E. 2000, ApJ, 534, 277

Winkler, C., Courvoisier, T. J.-L., Di Cocco, G., et al. 2003, A\&A, 411, L1

Worrall, D. M., Marshall, F. E., Boldt, E. A., \& Swank, J. H. 1982, ApJ, 255, 111

Yamauchi, S., Ebisawa, K., Tanaka, Y., et al. 2009, PASJ, 61, 225 


\section{Appendix A: Background model}

The background model used in K07 was slightly changed in this study. Since the GLS observations were performed over a relatively short time period, we removed the long-term time part from the equation. Instead of using the gain parameter to trace orbital modulations of the background rate, we used the spacecraft orbital phase $P$ in a quadratic polynomial form. Hereafter, we consider only the detector count rates after removal of the contribution of point sources. The model of the detector background $25-60 \mathrm{keV}$ count rate, $D_{\text {bgd }}$, is made of a linear combination of the $600-1000 \mathrm{keV}$ detector count rate, $H$, and phase

$D_{\text {bgd }}=$ const. $+a H+b_{1} P+b_{2} P^{2}$.

The coefficient $a$ was calculated using observations pointed away from the Galactic plane $\left(|b|>20^{\circ}\right)$ where the GRXE is not expected to be observed (see Table 1). The constant term and $b$ coefficients were determined individually for each spacecraft orbit from the observations at $|b|>20^{\circ}$, thus adjusting the model to the current background level. In fact, the constant term in Eq. (A.1) contains contribution from CXB and unknown intrinsic detector background. The last is also variable, which is traced by variability of this constant with time. In the current observations, its absolute value varies in the range of 800-900 mCrab from orbit to orbit, while permanent $\mathrm{CXB}$ contribution is expected to be at level of $550 \mathrm{mCrab}$ (Appendix B). Finding the difference between the observed and predicted by Eq. (A.1) detector count rate should yield the GRXE excess in the Galactic plane.

The detector count rate was converted to the convenient units of Crab flux, with $1 \mathrm{mCrab}=7.25 \times 10^{-6} \mathrm{cts} / \mathrm{s}$ in the $25-60 \mathrm{keV}$ band per IBIS FOV. The conversion coefficient was determined from observations of the Crab nebula in 2010 (Table 1). A flux of $1 \mathrm{mCrab}$ in the 25-60 keV energy band corresponds to $9.7 \times$ $10^{-12} \mathrm{erg} \mathrm{s}^{-1} \mathrm{~cm}^{-2}$ for a source with a Crab-like spectrum, $10.0 \times$ $E_{\mathrm{keV}}^{-2.1}$ phot $\mathrm{cm}^{-2} \mathrm{~s}^{-1} \mathrm{keV}^{-1}$.

\section{Appendix B: CXB cosmic variance}

The CXB emission coming from the population of unresolved extragalactic sources (active galactic nuclei, AGNs) is subject to Poissonian variations in the number of sources, intrinsic source variability, and nearby large-scale structure (see e.g. Fabian \& Barcons 1992). Here, we estimate the systematic limitations to the measured GRXE flux caused by CXB variations.

Using the extragalactic $\log N-\log S$ relation from Krivonos et al. (2010b) and following Revnivtsev et al. (2008), we estimated the relative uncertainty of the CXB flux in the $25-60 \mathrm{keV}$ band as

$\left(\frac{\delta I_{\mathrm{CXB}}}{I_{\mathrm{CXB}}}\right)_{\Omega} \sim 5.5 \times 10^{-2} S_{\max , 11}^{1 / 4} \Omega_{\mathrm{deg}}^{-1 / 2}$,

where $S_{\text {max } 11}$ is the maximum flux of undetected sources in units of $10^{-11} \mathrm{erg} \mathrm{s}^{-1} \mathrm{~cm}^{-2}$, and $\Omega_{\mathrm{deg}} \approx 286$ is the effective solid angle of the IBIS telescope. We adopted the CXB intensity to be equal to $1.89 \times 10^{-11} \mathrm{erg} \mathrm{s}^{-1} \mathrm{~cm}^{-2} \mathrm{deg}^{-2}$, based on the CXB spectrum model of Gruber et al. (1999) and the $\sim 10 \%$ higher normalization measured by INTEGRAL (Churazov et al. 2007). Using the limiting flux of the survey Eq. (B.1) yields a CXB variance at the level of $\sim 0.4 \%$. The absolute value is $2.3 \mathrm{mCrab}$ assuming a $\sim 550$ mCrab CXB flux per IBIS FOV. In the current work, we consider the CXB variance for an area of three IBIS FOVs, which approximately corresponds to the effective area of the GA survey.

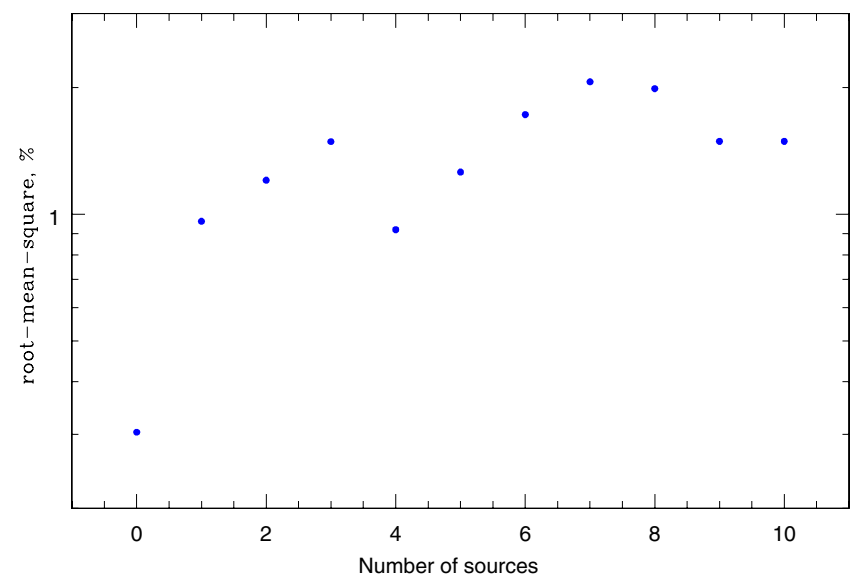

Fig. C.1. Relative root-mean-squared value of the detector rate as a function of number of sources in the FOV.

\section{Appendix C: Uncertainty in GRXE measurements}

The uncertainty of the background model, i.e. the accuracy of the ISGRI background rate prediction, is subject to statistical and systematical errors. The former can be easily estimated from the total number of counts $\left(\sim 3 \times 10^{5}\right)$ in the $25-60 \mathrm{keV}$ energy band per typical $S c W(\sim 2 \mathrm{ks})$.

The additional statistical effect is related to the IROS procedure, when the count rate attributed to a given source is removed from the detector using the known aperture function of the mask. To a first approximation, the total number of counts, $S$, associated with a given source is determined as the difference between the number of counts, $D_{1}$, in the detector pixels illuminated by the source through the mask and the number of counts, $D_{0}$, in the detector pixels blocked by the mask: $S=D_{1}-D_{0}$. The total flux on the detector is $D=D_{0}+D_{1}$. Thus, subtracting the contribution of the source yields the residual detector flux $D^{\prime}=D-S=2 D_{0}$. For a weak source in the center of the field of view, $D_{0} \approx D_{1} \approx 1 / 2 \times D$ and therefore the relative statistical uncertainty of measuring the detector count rate $\frac{D^{\prime}}{\sqrt{2 D_{0}}}=\sqrt{2} \frac{D}{\sqrt{D}}$ increases by a factor of $\sqrt{2}$. In practice, a more complicated model of a point source (see K07), implemented in the source subtraction algorithm, causes an even larger increase of statistical uncertainties.

To estimate the IROS induced uncertainty as a function of number of sources in the FOV, we studied a set of 22 consecutive observations without cataloged and detected sources (orbit 973, $S c W$ s 58-80). The relative standard deviation of detector count rate, as a function of the number of simulated sources, $N_{\mathrm{src}}$, is shown in Fig. C.1. The first point at $N_{\text {src }}=0$, RMS $=0.3 \%$, reflects the relative statistical scatter of the data. As seen from Fig C.1, the scatter rapidly increases with inclusion of sources in the FOV. A typical scatter of $\sim 1.0 \%$ on the $S c W$ time scale is observed in real data, as demonstrated in Sect. 4.

To estimate the systematic uncertainty of the method, we defined coefficients of the background model in Eq. (A.1) using the high-latitude observations (see Table 1) in the South Galactic hemisphere $(700 \mathrm{ks})$ and applied it to the North $(5.3 \mathrm{Ms})$. The non-existent GRXE flux was averaged over a given INTEGRAL orbit divided into the three equal intervals having three parts: one in the middle and two adjacent, see Fig. C.2 for reference. The middle part of each interval (in red) was supposed to have GRXE flux, and the neighboring parts (in blue) were used to correct the constant term (Eq. (A.1)). This set-up mimics the GLS pattern of observations. 
R. Krivonos et al.: Constraints on the ridge emission from the Milky Way anticenter

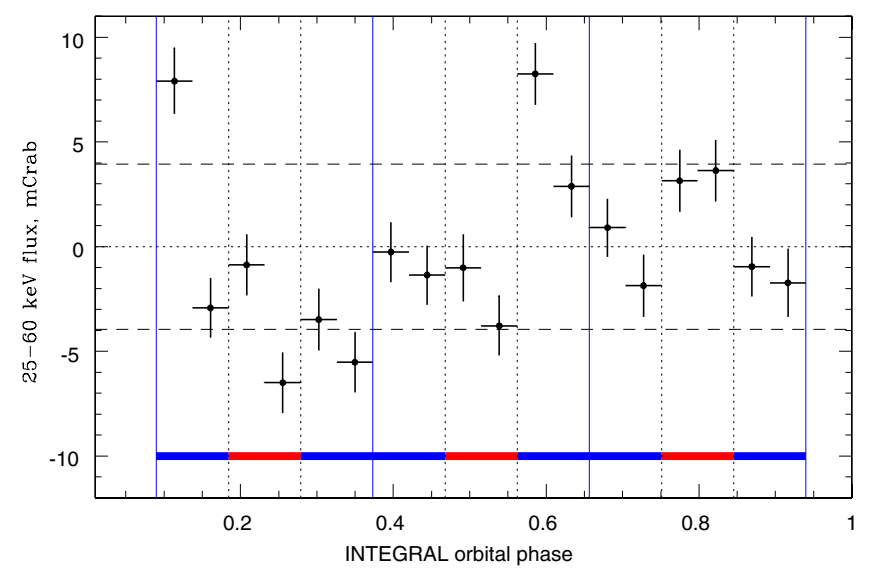

Fig. C.2. Residuals after subtracting the model-predicted count rate from the observed count rate for the $5.3 \mathrm{Ms}$ high-latitude observations averaged over spacecraft orbital phase. The black dashed lines represent a $1 \sigma$ deviation $(4.0 \mathrm{mCrab})$ of the averaged values from zero. Blue and red regions denote different phase intervals used for background model calibration and actual measurements, respectively.

The standard deviation of residuals from zero represents the systematic uncertainty of our background model, which is found to be $\sim 4.0 \mathrm{mCrab}$. The $25-60 \mathrm{keV}$ detector count rate increased from $\sim 2.2$ to $\sim 2.5$ Crab over the considered time period, hence the relative accuracy of the model is $\sim 0.17 \%$ of the observed background rate.
We summarize all the discussed uncertainties related to the GRXE measurements in the $25-60 \mathrm{keV}$ energy band. The values below are presented with respect to the background rate, which is assumed to be 2.5 Crab. Statistical uncertainties:

- $0.18 \%$ (4.5 mCrab) - count statistics, expected for $3 \times$ $10^{5}$ counts per typical $\mathrm{ScW}(\sim 2 \mathrm{ks})$,

- $0.30 \%$ (7.5 mCrab) - count statistics, observed in a typical $\mathrm{ScW}$ without any sources in the FOV. Since this value differs from the expected, it cannot be fully statistical. Some unexplored systematics or background variability can contribute to the scatter of the observed detector count rate.

- $1.00 \%$ (25 mCrab) - observed in a typical ScW with several sources in the FOV, related to the IROS procedure.

These uncertainties constitute the error of measurement and, naturally, decrease with increasing exposure. For example, the largest error of $25 \mathrm{mCrab}$ decreases to $1 \mathrm{mCrab}$ for a total exposure of 1 Ms. Systematic uncertainties:

- $0.16 \%$ (4.0 mCrab) - root-mean-squared residuals after background model subtraction from the observed count rate (Fig. C.2),

- $0.09 \%$ (2.3 mCrab) - CXB variance per IBIS FOV,

- $0.16 \%$ (4.0 mCrab) - CXB variance per GA survey area.

The systematics quadratically add to the error of the measurement. The total systematic error owing to the background model and $\mathrm{CXB}$ variance (per GA survey area) is $5.7 \mathrm{mCrab}$. 